\title{
DEPASSIVATION OF Si-SiO2 INTERFACE FOLLOWING RAPID THERMAL ANNEALING
}

\author{
Hao Jin, K.J.Weber, and A.W.Blakers \\ Centre for Sustainable Energy Systems, Faculty of Engineering and Information Technology, The Australian National \\ University, Canberra ACT 0200, Australia, email hao.jin@anu.edu.au
}

\begin{abstract}
The thermal stability of the $\mathrm{Si}_{-} \mathrm{SiO}_{2}$ interface of thermally oxidised silicon wafers is investigated using the Quasi-steady state photoconductivity decay (QSS-PCD) method. Planar silicon (100) and (111), as well as textured (100) wafers with various surface orientations were subjected to Rapid Thermal Annealing. Wafers textured with inverted pyramids displayed the most rapid depassivation rate, while (100) planar wafers showed the slowest depassivation rate. The depassivation rate of wafers which had been textured with inverted pyramids and subsequently rounded by acid etching was between that of (100) planar and wafers textured with inverted pyramids. The results suggest that the $\mathrm{Si}-\mathrm{SiO} 2$ interface on planar (100) surfaces is particularly thermally stable, and that the stability gradually decreases as one moves from from a (100) to a (111) surface orientation. The results also suggest that textured surfaces have a lower thermal stability, and a higher recombination rate, that planar surfaces of the same area and surface orientation.
\end{abstract}

\section{INTRODUCTION}

Thermally grown oxide layers are commonly used to reduce the silicon interface state density for passivation of the surfaces of solar cells due to the low achievable interface state densities and low recombination velocities of the $\mathrm{Si}-\mathrm{SiO}_{2}$ interface. If an antireflection coating is applied on top of a thin layer of $\mathrm{SiO}_{2}$, such as a layer of silicon nitride deposited by low pressure chemical vapor deposition, then the $\mathrm{Si}-\mathrm{SiO}_{2}$ interface is exposed to high temperatures during the deposition process which can result in depassivation of the interface.

It is known that the interface properties depend, among other factors, on the crystal orientation. In general, (111) oriented surfaces are more difficult to passivate than (100) surfaces. (111) surfaces always display a larger value for the interface trap density $\mathrm{D}_{\text {it }}$ than (100) surfaces [1,2]. Texturing provides good optical properties for solar cells but may decrease the achievable degree of surface passivation by increasing the surface area and by exposing crystal planes that are inherently more difficult to passivate.

We investigated the thermal stability of the $\mathrm{Si}-\mathrm{SiO}_{2}$ interface for different surface orientations. In order to investigate a range of surface orientations, some (100) wafers were textured using an alkali solution to form inverted pyramids. Some of the textured wafers were then etched in acid solution for 3 or 9 minutes to form surface orientations between (111) and (100).

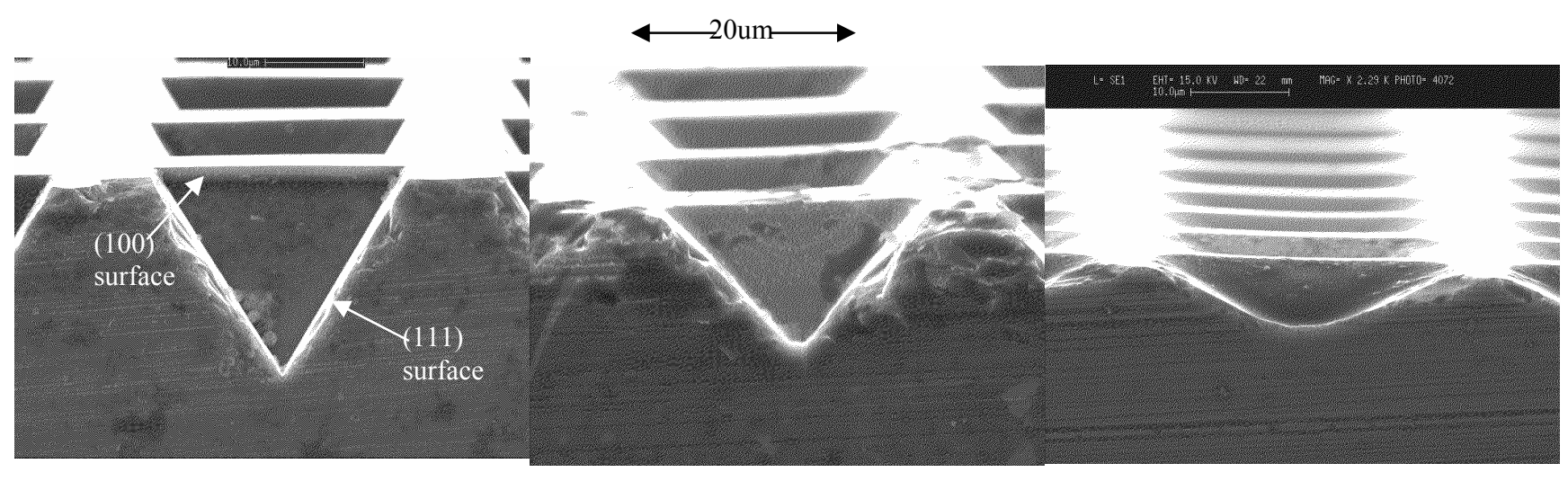

Fig 1. SEM picture of cross section of the etched dote structure 


\section{EXPERIMENTAL DETAILS}

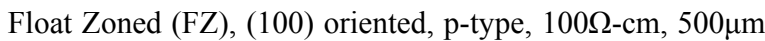
thick wafers and (111) oriented, n-type, $100 \Omega-\mathrm{cm}, 500 \mu \mathrm{m}$ thick wafers were taken as the starting materials. They were initially etched in 10:1 $\mathrm{HNO}_{3}$ : $\mathrm{HF}$ solution until shiny. Some (100) wafers were textured to form inverted pyramids. Some of these wafers were subsequently etched in $\mathrm{HF}: \mathrm{HNO}_{3}$ solution for 3 or 9 minutes. SEM pictures of the cross section for these structures are shown in fig 1. Etching reduces the inverted pyramid depth and results in a much shallower texture, with crystal planes between (111) and (100).

After a standard RCA cleaning, selected wafers were passivated with a light phosphorous diffusion ( $R \sim 360$ $\mathrm{ohm}$ /square after thermal drive in), a thermally grown oxide $(\sim 50 \mathrm{~nm})$ at $1000^{\circ} \mathrm{C}$ in dry oxygen with an in-situ $\mathrm{N}_{2}$ anneal and a 30 minute anneal at $400^{\circ} \mathrm{C}$ in forming gas $(5 \% \mathrm{H} 2$ in argon). Isothermal and isochronal annealing was performed in high flow dry nitrogen by Rapid Thermal Annealling (RTA) to test the thermal stability of these structures.

Minority carrier lifetime measurements were made using the quasi-steady state photoconductivity decay (QSS-PCD) technique. For diffused samples, values for the emitter saturation current density $\mathrm{J}_{\mathrm{oe}}$ were measured at an injection level of $5 \times 10^{15} / \mathrm{cm}^{2}$. On undiffused samples, the effective surface recombination velocity $\mathrm{S}_{\text {eff }}$ was estimated from the effective lifetime using PC-1D modeling, assuming a very high bulk lifetime $(30 \mathrm{~ms})$. This allowed derivation of an empirical equation relating the measured minority carrier lifetime at a particular injection level to the effective surface recombination velocity, $S_{\text {eff }}$, valid for $S_{\text {eff }}$ values of no more than $\sim 7000 \mathrm{~cm} / \mathrm{s}$. The $S_{\text {eff }}$ was estimated for the highest measured effective lifetime at in the injection range of $1 \times 10^{14} / \mathrm{cm}^{2}$ to $2 \times 10^{14} / \mathrm{cm}^{2}$. This estimation can be applied to our samples if the effective lifetime is greater than $10 \mu \mathrm{s}$.

\section{RESULTS AND DISCUSSION}

Fig 2 and Fig 3 show the surface depassivation process of both undiffused and phosphorus diffused samples. Isothermal anneals were done at $550^{\circ} \mathrm{C}$ from 30 seconds to 360 seconds in nitrogen. On all samples, the rate of increase of $\mathrm{S}_{\text {eff }}$ or $\mathrm{J}_{\mathrm{oe}}$ decreases with increasing anneal time. The (100) surface shows the lowest surface recombination velocity and depassivation rate, while textured wafers display the most rapid depassivation. The depassivation rates of the etched samples are between those of the textured samples and the (100) samples. The (111) surface has a depassivation rate similar to that of the sample that was etched for 3 minutes following texturing.

Figures 4 and 5 show the isochronal RTA results. All samples were annealed for 3 minutes in nitrogen. For all the samples, the depassivation rate increases with annealing temperature. Similar to Fig 3 and Fig 4, (100) samples display the slowest isochronal depassivation rate while textured wafers display most rapid depassivation. (111) samples perform similar to the 3 min etched samples.
It is clear that surface orientation plays an important role in determining the surface recombination properties, with (111) surfaces being much more difficult to passivate than (100) surfaces. This may be partly due to a higher defect density at oxidised (111) surfaces, but it appears to be, at least in part, also the result of increased recombination activity of defects at (111)

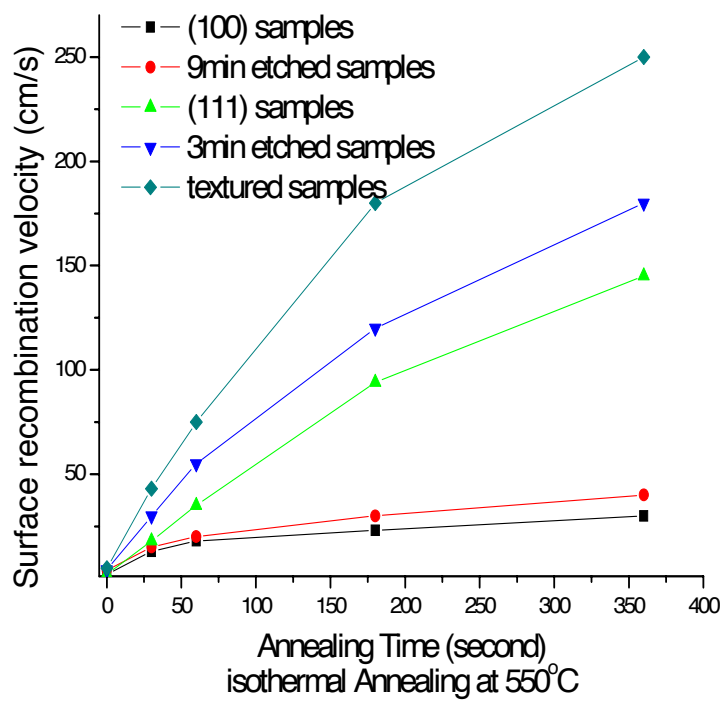

Fig 2: Effective surface recombination velocity $\mathrm{S}_{\text {eff }}$ vs isothermal annealing time for RTAs

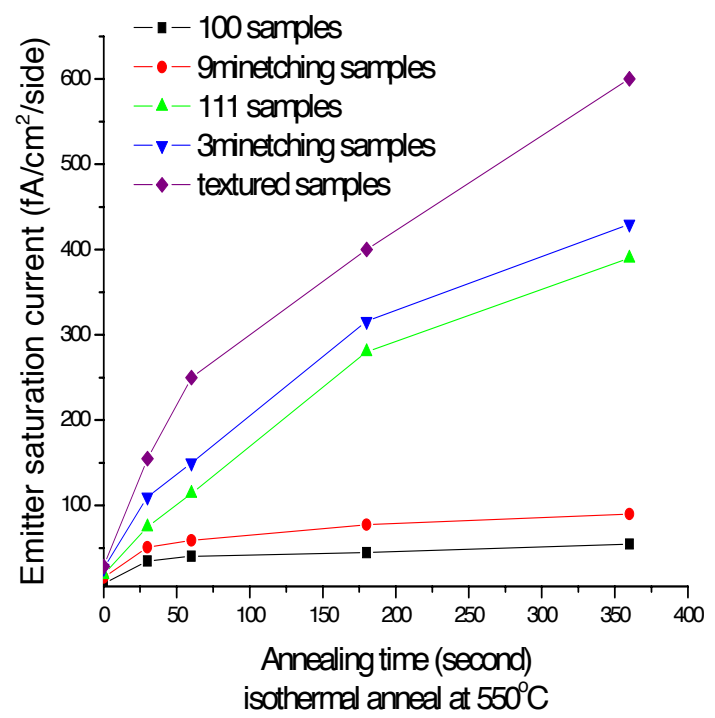

Fig 3: Emitter saturation current (Joe) vs isothermal annealing time for RTAs

surfaces. As another example, in a separate experiment diffused, oxidised (100) and (111) wafers were vacuum annealed at $800^{\circ} \mathrm{C}$ for $2 \mathrm{hrs}$ to remove all hydrogen from the interface. After this treatment, the $\mathrm{J}_{\mathrm{oe}}$ of the (111) wafers was an order of magnitude larger than that of the (100) samples while the 
difference in defect densities between (111) and (100) surfaces is typically 'only' about a factor of 3 .

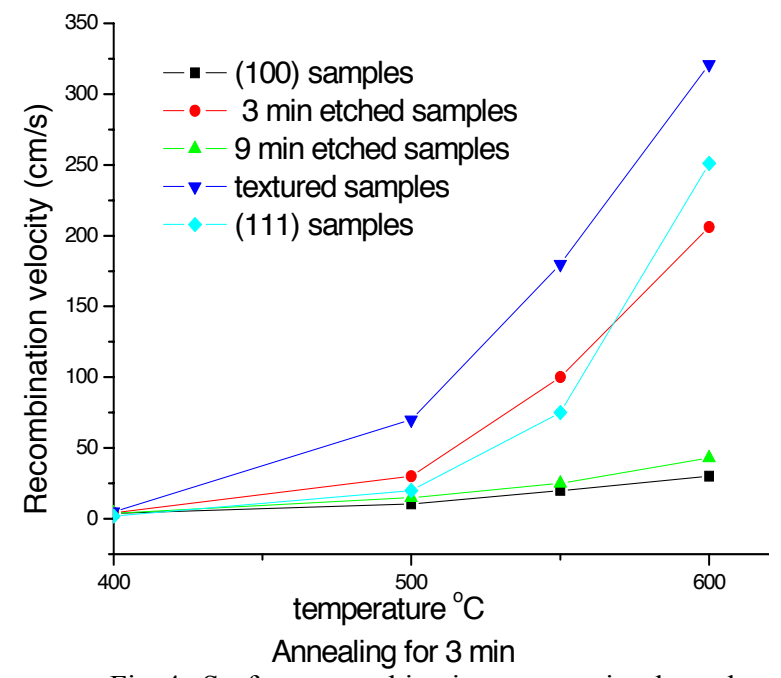

Fig 4: Surface recombination rate vs isochronal annealing

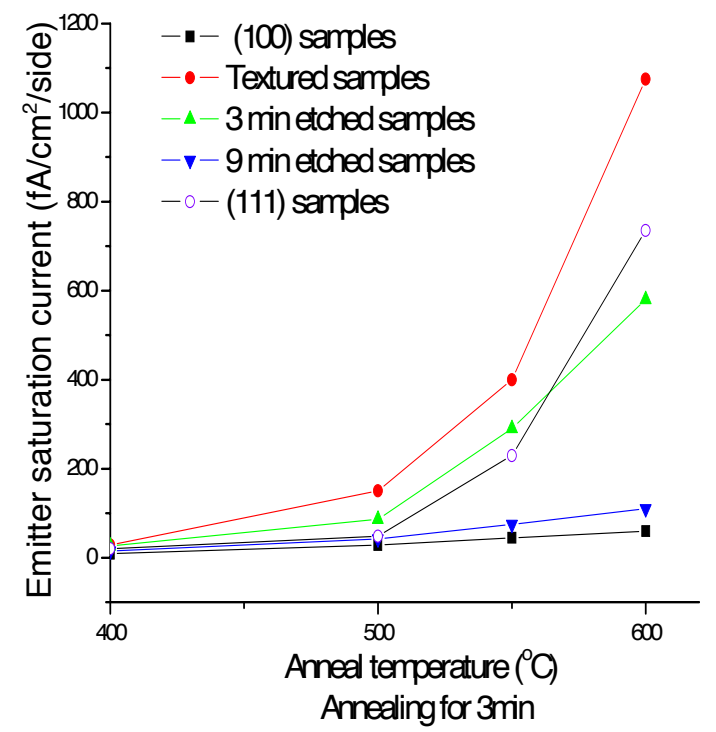

Fig 5: Emitter saturation current vs isochronal annealing

An important point to note is that while the overall surface area of the sample with inverted pyramids is obviously larger than that of the planar samples, the increase in surface area is not large (approximately $25 \%$, since only less than a third of the surface is textured), and the area of (111) surface is actually less than that of the planar (111) samples. Thus, the behaviour of the textured samples cannot be explained by an increase in surface area. Another possible reason could be that the textured surfaces are more perfect (111) surfaces than those of (111) planar wafers, which ordinarily have a tolerance on the surface orientation of $+/-0.5$ degrees. The etching may introduce further changes at the atomic scale to these surfaces. However, it is surprising that even the textured wafers that were subsequently acid etched for 3 minutes display a behaviour similar to that of the (111) planar samples.

\section{CONCLUSION}

RTA and QSSPCD measurements were applied to test the thermal stability of different Si surface orientations. During isochronal and isothermal anneals, textured samples displayed the fastest depassivation rate. Surface orientations between textured surfaces and (100) surfaces showed a slower depassivation rate than the textured surface but a higher rate than the (100) surface. As the texture becomes more and more shallow, resulting in surfaces with orientations closer to (100), the depassivation rate gradually decreases and approaches that of (100) surfaces.

\section{REFERENCES}

1. P.K.Hurley, B.J.O'Sullivan, F.N.Cubaynes, P.A.Stolk, F.P.Widdershoven and J.H.Das, J. Electrochemical Soc., 149, G194, 2002

2. A. Stesmans and V.V. Afanas'ev, J. Vac. Sci. Technol. B, 16, 3108 (1998) 